\title{
Surgical ventricular reconstruction in mice: Elucidating potential targets for combined molecular/surgical intervention
}

\author{
Che-Chung Yeh, PhD, Deepak Malhotra, MD, Hongzhe Li, MD, PhD, Susan Nicholas, MD, Richard Tu, MD, and \\ Michael J. Mann, MD
}

\begin{abstract}
Objectives: We hypothesize that persistent alterations in molecular signaling may drive recurrent pathologic remodeling even after the reduction of mechanical stress achieved via surgical ventricular reconstruction. We developed a murine model of surgical ventricular reconstruction that would facilitate molecular analysis of the postreconstruction myocardium and allow future exploitation of genetic models.
\end{abstract}

\begin{abstract}
Methods: C57/B6 mice underwent coronary artery ligation. For surgical ventricular reconstruction at 4 weeks after myocardial infarction, a purse-string suture (7-0 polypropylene) achieved at least partial exclusion of the apical aneurysm. Serial echocardiography was correlated to measurements of apoptosis and to Western blot analysis of key signaling cascades.
\end{abstract}

Results: An immediate $21.7 \% \pm 2.6 \%$ improvement in fractional shortening was seen in the remaining myocardium after surgical ventricular reconstruction. Reduction in left ventricular volume and improved function persisted at 1 week, but recurrent dilatation at 4 weeks (left ventricular end-diastolic volume of $63.5 \pm 2.5$ vs $42.1 \pm 5.4 \mu \mathrm{L}$ immediately after reconstruction; $P<.05$ ) was associated with a loss of functional improvement (fractional shortening $41.2 \% \pm 2 \%$ vs $46 \% \pm 0.9 \% ; P<.01$ ). At 1 week after surgical ventricular reconstruction, there was a transient reduction in myocardial apoptosis. A steady reduction in cardioprotective myocardial Akt activation, however, was not affected by ventricular reconstruction.

Conclusion: This murine model recapitulates both the immediate benefits of surgical ventricular reconstruction and the longer-term recurrence of dilated cardiomyopathy seen previously in some animal models and human studies. Early analysis has begun to implicate persistent signaling changes in the postinfarction myocardium that may be responsible for recurrent dilatation after surgical ventricular reconstruction and that may become targets for combined surgical and molecular interventions.

Current management of congestive heart failure primarily slows progression of disease. Surgical treatments have been proposed to improve the biophysics of the dilated left ventricle (LV) and to reduce the stimulus for ongoing pathologic remodeling. ${ }^{1}$ Early studies of surgical ventricular reconstruction (SVR), and particularly of aneurysm resection, have demonstrated an improvement in both ventricular function and congestive heart failure symptoms. ${ }^{2-8}$ These studies, however, have also demonstrated recurrent LV dilatation, suggesting that a reduction in mechanical stress alone does not address all of the changes in myocardial biology that drive the remodeling process. $4,8,9$

Much has been learned regarding molecular signaling in the myocardium. A number of pathways have been identified that drive both ventricular hypertrophy and a transition to dilated cardiomyopthay. ${ }^{10}$ Genetic mouse models have re-

From the Division of Cardiothoracic Surgery University of California, San Francisco and VA Medical Center, San Francisco, Calif.

This study was supported by the American Heart Association, grant $0465090 \mathrm{Y}$ to M.J.M., and the National Institutes of Health grants 1K08HL079239-01 and 1R01 HL083118-01.

Received for publication July 24, 2008; revisions received Sept 3, 2008; accepted for publication Sept 12, 2008.

Address for reprints: Michael J. Mann, MD, Cardiothoracic Surgery, 415 Clement St,

112D, San Francisco, CA 94121 (E-mail: mannm@surgery.ucsf.edu).

J Thorac Cardiovasc Surg 2009;137:942-9

$0022-5223 / \$ 36.00$

Copyright (c) 2009 by The American Association for Thoracic Surgery

doi: $10.1016 /$ j.jtcvs. 2008.09 .038 vealed changes in even a single enzyme that can influence macroscopic ventricular structure and function. ${ }^{1-15}$ In particular, phosphotidyl inositol-3 kinase (PI3K)/Akt signaling can protect cardiac myocytes from apoptosis, ${ }^{11,16}$ although the pathway has been associated with both pathologic hypertrophy ${ }^{12}$ and adaptive, physiologic hypertrophy. ${ }^{11}$ Mitogenactivated protein (MAP) kinases also help determine cardiac myocyte and ventricular fate; p38 MAP kinase and Jun $N$ terminal kinase (JNK) have, in some studies, been associated with cardiac cell apoptosis and ventricular dilatation, ${ }^{13,14}$ whereas the MAP kinase ERK (extracellular signal-related kinase) may protect these cells. ${ }^{15}$

We describe a murine model of post-myocardial infarction (MI) heart failure and SVR that may help elucidate the molecular mechanisms of recurrent ventricular dilatation. We hypothesize that critical changes in myocardial signaling persist despite ventricular reconstruction (Figure 1) and that hybrid molecular and surgical interventions may sustain and even enhance benefit. We measured the activation of key elements of the PI3K/Akt and MAP kinase cascades in post-MI hearts subjected to either SVR or sham reoperation.

\section{MATERIALS AND METHODS \\ Mouse Coronary Ligation and SVR \\ Male C57/B6 mice (25 g) were anesthetized with $1.5 \%$ inhaled isoflur- ane at 115 breaths/min. A left lateral thoracotomy incision at the fourth in- tercostal space exposed the LV and atrial appendage. Although mouse}




\section{Abbreviations and Acronyms \\ $\mathrm{EF} \quad=$ ejection fraction \\ ERK = extracellular signal-related kinase \\ FS $=$ fractional shortening \\ JNK $=$ Jun $N$-terminal kinase \\ $\mathrm{LAD}=$ left anterior descending coronary artery \\ $\mathrm{LV}=$ left ventricle \\ LVEDV $=$ left ventricular end-diastolic volume \\ LVESV $=$ left ventricular end-systolic volume \\ MAP = mitogen-activated protein \\ MI = myocardial infarction \\ PI3K = phosphotidyl inositol-3 kinase \\ SVR = surgical ventricular reconstruction \\ TUNEL $=$ terminal deoxynucleotidyl transferase- mediated dUTP nick end labeling}

coronary anatomy differs from that of the typical human heart, there is generally at least one large branch of the left main coronary artery on the anterior wall of the LV. A 7-0 polypropylene suture was used to ligate this vessel, which, for convenience, we refer to as the left anterior descending artery, approximately one third the distance from the base to the apex. Preliminary studies documented a reproducible MI of $30 \%$ to $40 \%$ of the LV as assessed by computerized image analysis (Scion Corp, Frederick, Md). A sodium hyaluronate-carboxymethyl cellulose membrane patch (Seprafilm; Genzyme Corporation, Cambridge, Mass) was placed between the LV and chest wall to facilitate reoperation. SVR 4 weeks after MI involved placement of a purse-string suture (7-0 polypropylene) around the infarcted/ aneurysmal region. Sham operation consisted simply of redo thoracotomy. A total of 56 mice with left anterior descending artery ligation were randomly divided into (1) sham control (20 mice) and (2) SVR (36 mice). Mortality from thoracotomy was less than $10 \%$ and approximately $20 \%$ with SVR. Significant morbidity was limited primarily to failure to thrive after MI. Diastolic arrest was induced by high potassium injection at the time the animals were humanely killed. All procedures conformed with the "Guide for the Care and Use of Laboratory Animals" published by the National Institutes of Health (NIH Publication No. 85-23, revised 1996) and were approved by the Institutional Animal Care and Use Committee of the San Francisco Veterans Affairs Medical Center.

\section{Echocardiography}

Transthoracic echocardiography was performed on awake, minimally restrained mice with an Acuson Sequoia 512 machine (Siemens AG, Berlin, Germany) and a 13-MHz probe. A 2-dimensional short-axis view of the LV was obtained at the level of the papillary muscles, and M-mode tracings were recorded. LV fractional shortening (FS) was calculated as LV diastolic dimension - LV systolic dimension. ${ }^{17,18}$ Measurements were made before and immediately after SVR and at 1, 2, and 4 weeks.

\section{Histology and Apoptosis}

Mouse hearts arrested in diastole were pressure-fixed in formalin and paraffin-embedded. Serial sections $(5 \mu \mathrm{m})$ were stained with Gomori trichrome to identify fibrillar, collagen-rich scar. For apoptosis, thin sections underwent TUNEL (terminal deoxynucleotidyl transferase-mediated dUTP nick end labeling) staining (Chemicon, Temecula, Calif) and apoptotic indices were calculated in 3 sections per heart. Ligase staining (ApopTag. Peroxidase ISOL Kit, Chemicon) of selected adjacent sections was used to confirm the specificity of TUNEL.

\section{Protein Expression Analysis}

Hearts were divided into infarct/border zone (defined as the translucent infarct tissue plus the immediate $\sim 1 \mathrm{~mm}$ of surrounding myocardium) and remote, uninfarcted myocardium and were homogenized in a lysis buffer $(0.13 \mathrm{~mol} / \mathrm{L} \mathrm{KCl}, 1 \mathrm{mmol} / \mathrm{L}$ ethylenediaminetraacetic acid, $1 \mathrm{mmol} / \mathrm{L}$ ethyleneglycol tetraacetic acid, $1 \mathrm{mmol} / \mathrm{L} \mathrm{Na}_{3}$ (vanadate ion), $5 \mathrm{mmol} / \mathrm{L}$ sodium fluoride, $20 \mathrm{mmol} / \mathrm{L} N$-2-hydroxyethyl-1-piperazine- $N$-2-ethanesulfonic acid (HEPES), and protease inhibitor cocktail tablet (Roche Diagnostics, Mannheim, Germany). BCA Protein Assay Reagent Kit (Pierce, Rockford, Ill) was used to measure protein concentration. Equal amounts of protein were separated by NuPAGE Novex Bis-Tris Gels (Invitrogen Corporation, Carlsbad, Calif) and transferred to polyvinylidene fluoride membranes (Invitrogen Corporation). Blots were probed with antibodies specific for phosphorylated Akt and total Akt, phosphorylated ERK and total ERK, phosphorylated p38 and total p38, phosphorylated p70S6 kinase and total p70S6 kinase, Bcl-2, Bax, or BAD (Cell Signaling, Beverly, Mass) with appropriate horseradish peroxidase-conjugated antibodies as secondary antibodies (Cell Signaling). SuperSignal West Femto Maximum Sensitivity substrate (Pierce Biotechnology, Rockford, Ill) was used for visualization. Density analysis by Scion Image (Scion Corporation, Frederick, Md) was used to determine relative protein quantitification.

\section{Statistics}

Data are reported as mean \pm SEM. Comparisons between groups were made with the $t$ test and analysis of variance.

\section{RESULTS}

\section{SVR With Partial Aneurysm Exclusion}

Trichrome staining of a representative mouse heart immediately after SVR demonstrated the exclusion of the majority of postinfarct LV aneurysms, with subsequent alteration of LV chanber size and geometry (Figure 2). The inability to access the septal component of an infarct and excessive mortality with overly aggressive purse-string reductions in LV size resulted in only partial aneurysm exclusion in many animals (Figure 2).

\section{Immediate Effect of SVR on LV End-Diastolic Volume and Redilation}

Echocardiography confirmed similar LV end-diastolic volume (LVEDV), end-systolic volume (LVESV), and FS between the groups that were subsequently randomized to either SVR or sham control at 4 weeks after left anterior descending artery ligation. Immediately after SVR, we observed an anticipated reduction in LV dimensions (Figure 3, $A)$ and LVEDV $(42.1 \pm 5.4$ vs $69.0 \pm 5.8 \mu \mathrm{L} ; P<.01$; Figure $3, B)$. No differences were observed in LV volumes after sham operation. Serial echocardiography demonstrated that the LVEDV gradually increased after SVR (Figure 3,B) and that LVEDV in the post-SVR animals was no longer statistically different from that in the sham group, or pre-SVR levels, by 4 weeks after reoperation.

\section{Ejection Fraction (EF) and FS Status-Post SVR}

Echocardiography was chosen as a primary functional analysis because it may be the only modality capable of 


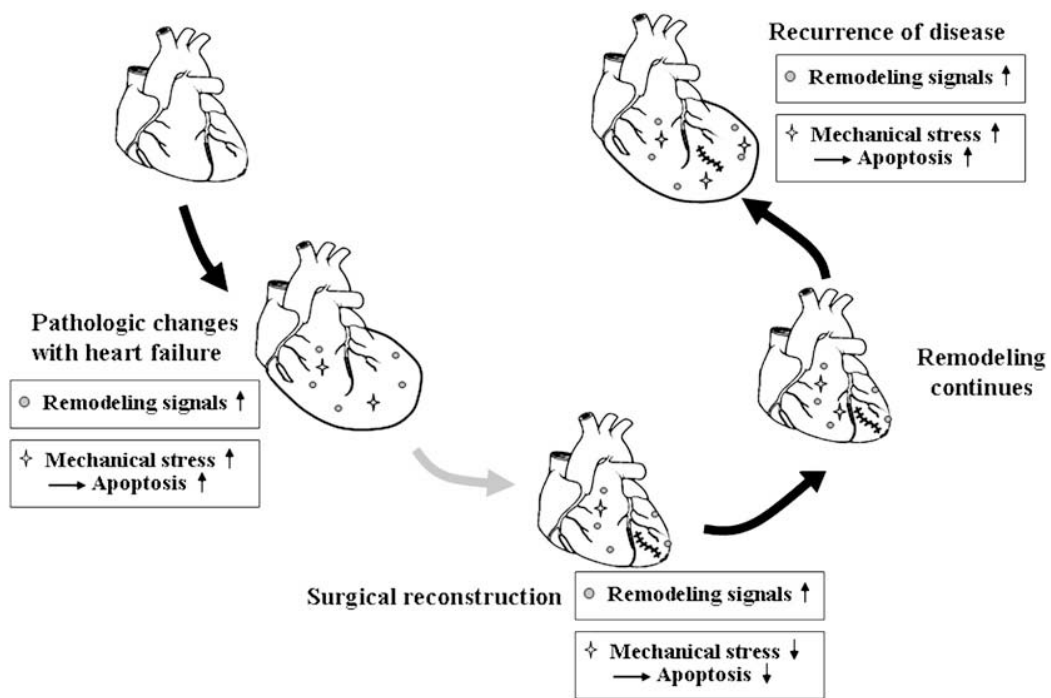

FIGURE 1. Hypothesized role of persistent pathologic signaling motif in the recurrence of ventricular dilatation after SVR. Immediate reduction in LV wall stress leads to a reduction in apoptotic stimuli, whereas persistent alterations in fundamental myocardial signaling pathways, such as PI3K/Akt and MAP kinase cascades, drive ongoing SVR. A return of ventricular dilatation and resultant wall stress leads to a return of apoptotic myocyte loss. SVR, Surgical ventricular remodeling; $L V$, left ventricular; $M A P$, mitogen-activated protein.

measuring cardiac function in awake, minimally restrained mice, thereby eliminating the profound impact of general anesthesia on cardiac function in rodents. Furthermore, it allowed serial measurements in the same animals, believed to be critical for this study of SVR. Focusing the analysis of FS at the level of the more easily identifiable mitral apparatus helped standardize the echocardiographic imaging, which was carried out in a blinded fashion. Echocardiographic evidence of improved hemodynamic function immediately after SVR was corroborated in a small number of hearts via ex vivo Langendorff preparation (data not shown).

Echocardiography revealed an improvement in ejection fraction (EF) after SVR that corresponded to the reduction in LV chamber size $(74.5 \% \pm 0.6 \%$ vs $66.9 \% \pm 1.4 \%$ before SVR; $P<.01)$. While a reduction in volume can directly lead to an improvement in EF, even more striking was an im- provement in FS $(21.7 \% \pm 2.6 \%$ improvement over preSVR) measured in the myocardial wall at the level of the proximal mitral apparatus, distant from the region of the infarct (Figure 4). These data suggest that an immediate reduction in LV wall stress allowed a functional improvement in more global myocyte contractility. By 4 weeks after SVR, however, serial echocardiography indicated a significant decline in both EF and FS (Figures 3, B, 4). Parameters of LV function in the sham controls remained relatively stable during this time period.

\section{Apoptosis and Expression of Apoptotic Regulators in the Post-SVR Ventricle}

Previous studies have suggested that LV dilatation is mediated at least in part through the loss of cardiac myocytes to programmed cell death, or apoptosis. We therefore
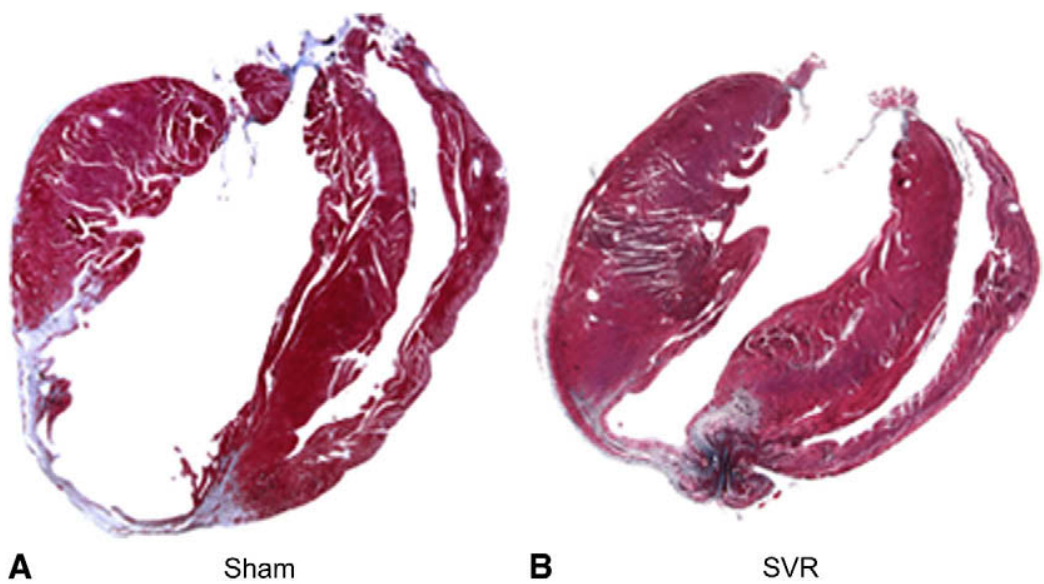

FIGURE 2. Gamori trichrome stain of mouse heart 4 weeks after myocardial infarction with (B) or without (A) surgical ventricular remodeling (SVR). 


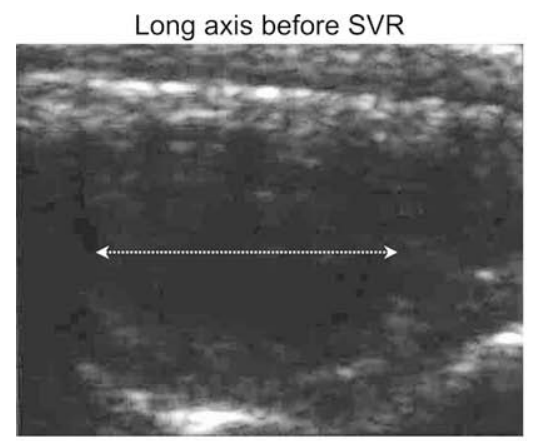

Short axis before SVR

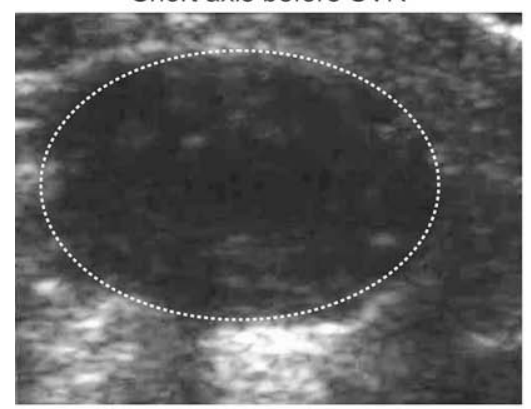

A

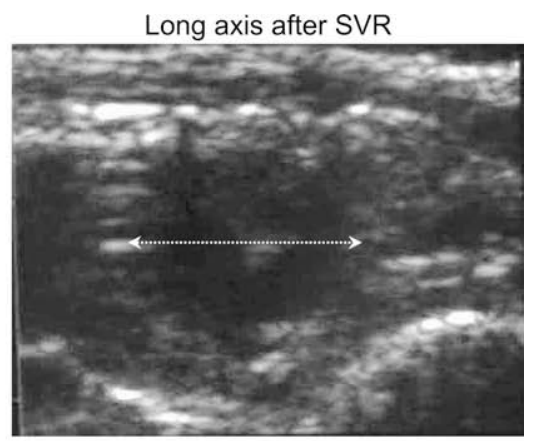

Short axis after SVR

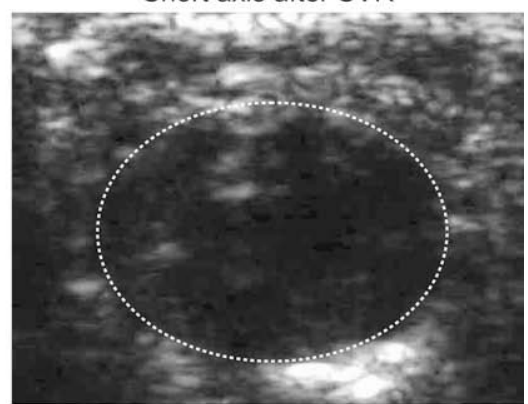

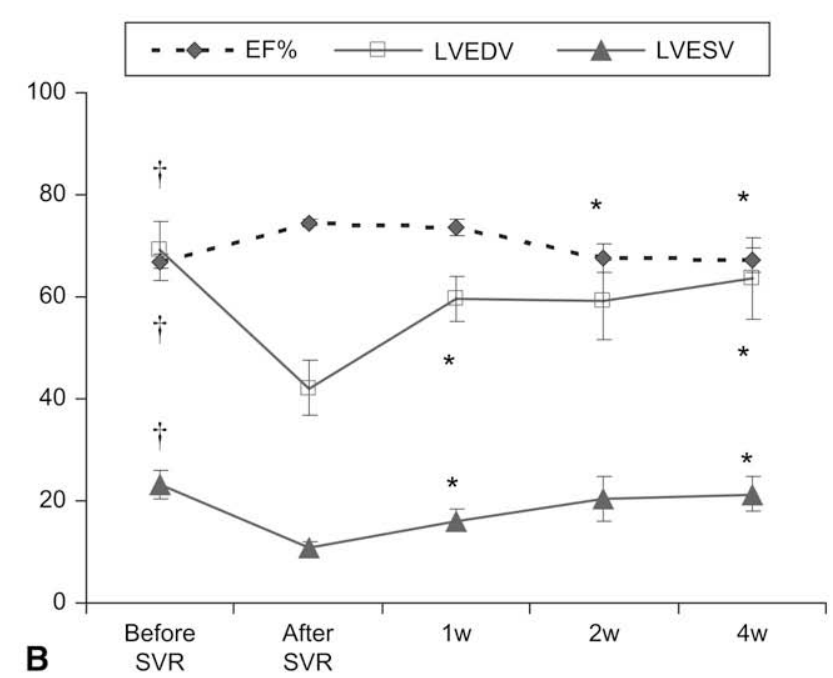

FIGURE 3. A, Echocardiograms of infarcted hearts before and immediately after SVR. SVR decreased both the long axial length and cross-sectional area of the LV chamber, as indicated by dashed lines. B, Time course of LVEDV, LVESV, and EF status-post SVR. The LVEDV, LVESV, and EF all improved immediately after SVR $(\dagger P<.01)$ but gradually returned to approximate pre-SVR levels $(* P<.05$ vs levels immediately after SVR) (n $=8) . E F$, Ejection fraction; $L V E D V$, left ventricular end-diastolic volume; $L V E S V$, left ventricular end-systolic volume; $S V R$, surgical ventricular reconstruction.

measured both rates of myocardial apoptosis and the protein expression levels of several key mediators of apoptosis in the post-SVR ventricle and compared them with those in sham controls (Figure 5, $A$ and $B$ ). The regulation of apoptosis is characterized by an intricate balance between proteins that play antiapoptotic and proapoptotic roles. Although levels of the antiapoptotic protein Bcl-2 did not change significantly between weeks 1 and 4 after reoperation, either in SVR or sham control animals, a decrease in expression of proapoptotic Bax resulted in an increase in the Bcl-2/Bax ra- tio at week 1 after reoperation compared with normal hearts (Figure 5, C). This increase was more pronounced in SVR versus sham controls $(P<.05)$. By week 4 , this ratio in both groups returned to levels seen in normal hearts. This early antiapoptotic signal was counterbalanced in sham controls by a drop in proapoptotic BAD phosphorylation (inactivation) at week 1 (Figure $5, D$ ). Interestingly, BAD phosphorylation remained at normal levels in SVR hearts at 1 week, at which time apoptotic indices were down. Early after reoperation, levels of antiapoptotic Bcl-XL expression 


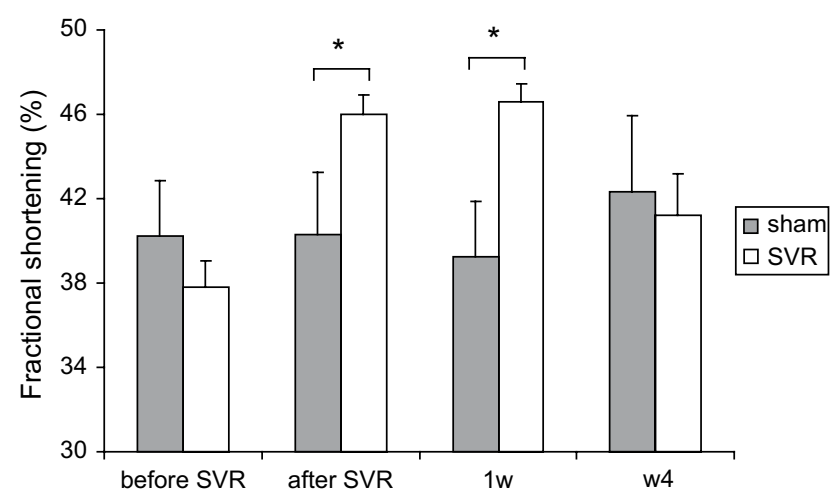

FIGURE 4. Changes in FS status-post SVR. An immediate improvement in FS in the SVR group persisted at week $1(* P<.05, \mathrm{n}=24-26)$ but not week 4 after SVR, whereas no significant change in FS was seen in the sham group $(\mathrm{n}=9) . F S$, Fractional shortening; $S V R$, surgical ventricular remodeling.

were decreased but then increased very significantly in both treatment groups at week 4 (Figure 5,E).

\section{Changes in Myocardial Signaling}

Compared with normal myocardium, post-MI sham control hearts displayed a progressive decrease in Akt activation (as reflected in the relative phosphorylation of Akt on Western blot, Figure 6, A), and this drop in cardioprotective signaling was not affected by SVR. A small increase was observed at week 1 after reoperation in phosphorylation (ie, activation) of MAP kinase p38 in sham controls, although, in general, levels of p38 phosphorylation did not vary substantially between SVR and sham controls (Figure 6, $B)$. Similarly, increases in ERK phosphorylation (ie, activation) were seen in both sham controls and SVR hearts at week 1 after reoperation, although there was a return to baseline ERK phosphorylation in SVR, but not sham, hearts at week 4 (Figure 6, C). Interestingly, a drop in phosphorylation (ie, activation) of JNK was observed only in sham control hearts at week 1, although phosphorylation of this so-called stress-activated kinase, associated in some studies with myocardial apoptotsis, was increased above baseline in normal hearts in both treatment groups at week 4 (Figure 6,D).

\section{DISCUSSION}

In our murine model, SVR improved cardiac function. These reconstructed ventricles, however, were susceptible to recurrent dilatation, and a reduction in function was observed as early as 4 weeks after reconstruction. These results are similar to the findings of Nishina and associates ${ }^{3,19}$ in a rat model of aneurysm exclusion and are reminiscent of longer-term changes documented in clinical studies of linear or patch ventricular repair. ${ }^{20-22}$

The complex integration of multiple signaling pathways is not yet well understood in the evolution of post-MI cardiomyopathy. However, studies have suggested a cardioprotec- tive role for the PI3K/Akt pathway. ${ }^{23,24}$ The drop we observed in Akt phosphorylation in the post-MI myocardium may therefore play an important role in the loss and dysfunction of myocardial tissue; this drop persisted even after an improvement in ventricular geometry achieved via SVR.

Only minor differences were observed between sham control and SVR hearts in terms of the phosphorylation of MAP kinases p38 and ERK early after reoperation. MAP kinase activation is generally upregulated after ischemic insults, although the role of this activation has been studied more extensively in the progression from pressure overload-induced hypertrophy to dilated cardiomyopathy. It is not clear what role, if any, the observed reduction in phosphorylation of JNK, generally considered, like p38, a "stress-induced" kinase, might have played in sham control hearts 1 week after reoperation, although this change was not observed in SVR hearts. JNK phosphorylation was then increased in both groups by week 4 .

SVR results in a reduction in ventricular volume and a decrease in chamber radius. According to LaPlace's law, these changes decrease wall stress. A reduction in wall stress reduces myocardial oxygen demand and enhances ventricular contraction, ${ }^{9}$ and there is an increase in the extent and velocity of systolic fiber shortening. This phenomenon was reflected in the increases in FS and EF observed in our murine model. In addition, SVR is likely associated with acute and subacute changes in hemodynamic parameters, which, in turn, are likely to affect both ventricular remodeling and associated changes in myocyte signaling. Future studies may be necessary to sort out the various stimuli for changes in cardiac myocyte gene expression and kinase activation to optimize human translation of these interventions.

Furthermore, a reduction in mechanical stress may have also contributed to the reduced level of myocardial apoptosis seen early after SVR, and that may have been mediated by an increase in the Bcl-2/ Bax ratio and a preservation of $\mathrm{BAD}$ phosphorylation. As suggested by the schema in Figure 1, persistent changes in molecular signaling, however, such as the reduction of Akt phosphorylation, may drive cardiac remodeling at the cellular level, even after SVR. Subsequent recurrence of LV dilatation could, in turn, instigate a return to pathologic levels of wall stress and to the vicious cycle of apoptotic cell loss and further progressive LV enlargement.

Current surgical treatments for ischemic cardiomyopathy may not yield optimal long-term outcomes. ${ }^{2,7}$ This study explored changes in myocardial signaling that might induce reverse remodeling or "physiologic hypertrophy" to complement the immediate improvement in ventricular geometry achieved by SVR. In addition, cell-based and tissue-engineering approaches may be combined with reconstruction. Numerous studies have demonstrated benefit from stem cell delivery to injured myocardium, ${ }^{25-27}$ and more recent reports have also described bio-artificial matrices that may provide 


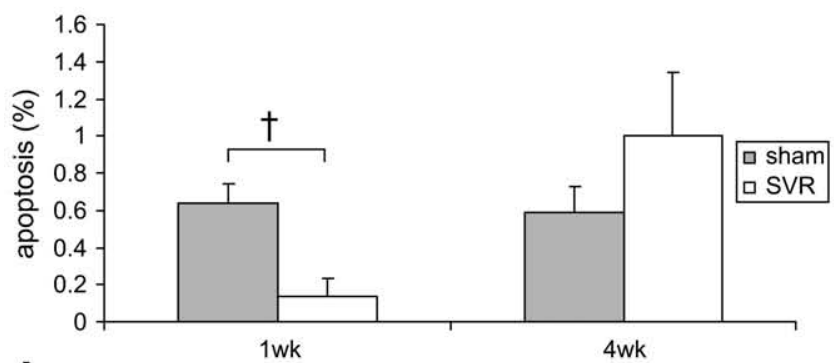

A
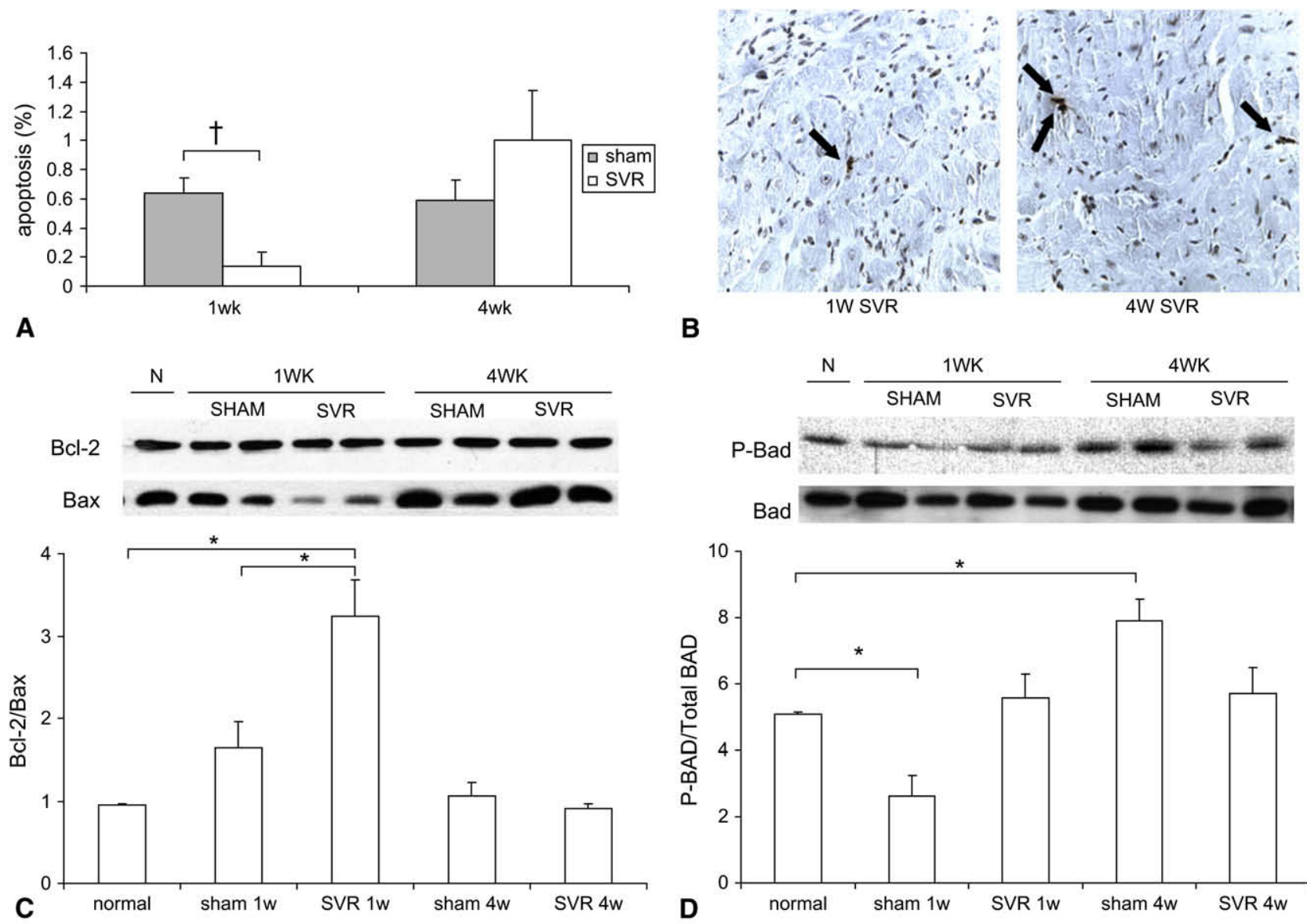

B
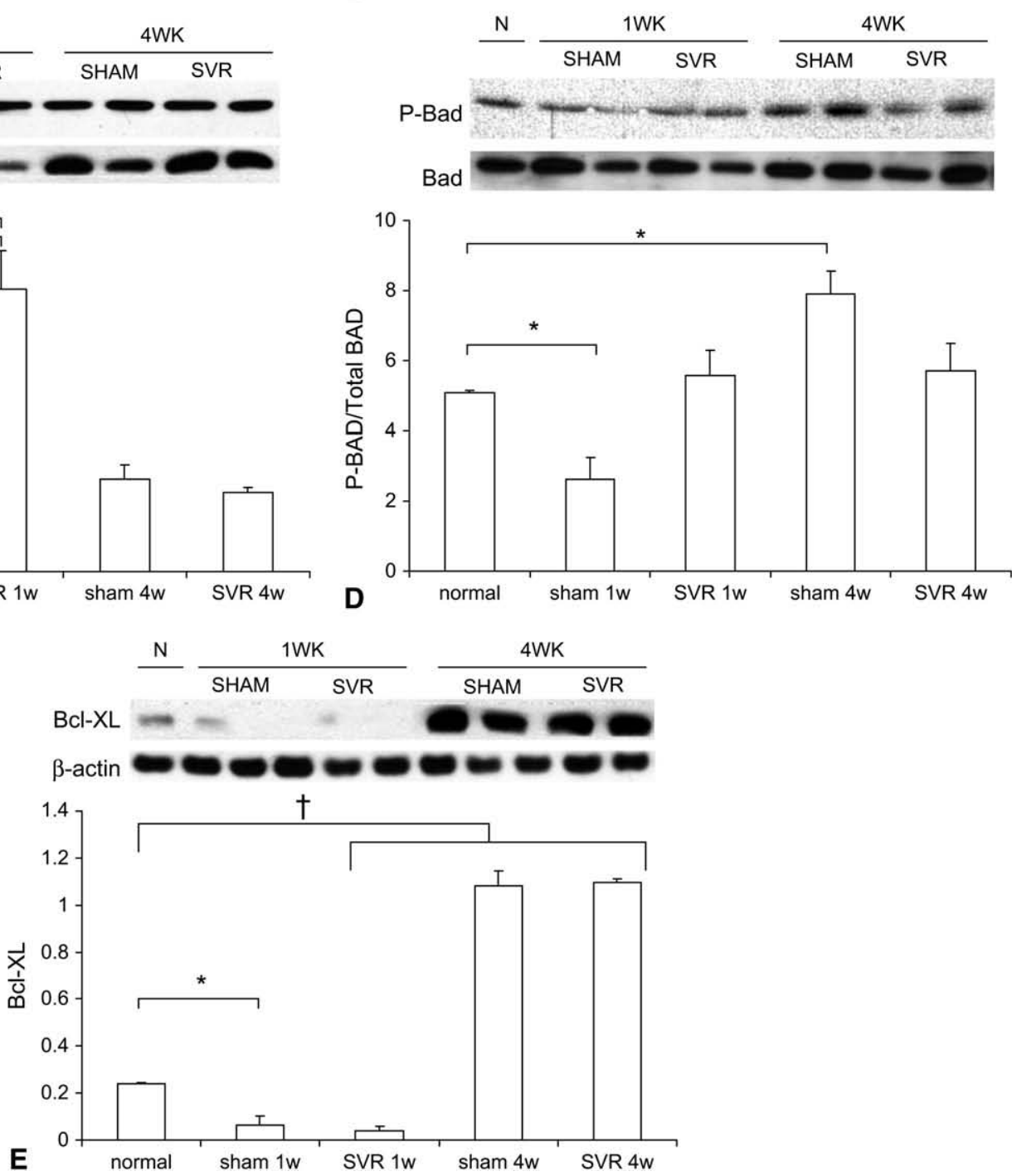

FIGURE 5. SVR effect on the survival of cardiomyocytes in the remote myocardium. Apoptotic indices derived from TUNEL staining (A) were lower at 1 week $(\dagger P<.01)$ but not at 4 weeks $(P=$ not significant) in SVR hearts compared with sham controls $(\mathrm{B})$. The ratio of expressions of Bcl-2/Bax $(\mathrm{C})$, expression of Bcl-XL (D), and phosphorylation (inactivation) of proapoptotic BAD (E) in sham control and SVR hearts at 1 and 4 weeks after reoperation. $(\dagger P<.01, * P<$ $.05, \mathrm{n}=3)$. SVR, Surgical ventricular reconstruction.

mechanical support to the injured myocardium and enhance myocardial regeneration. ${ }^{28,29}$ The direct access of the surgeon at the time of SVR may facilitate otherwise challenging, early applications of these approaches.
Like any small animal model, ours must be understood in light of its numerous limitations. We induced acute MI in the setting of otherwise normal coronary anatomy and function; more complex human disease may be better mimicked 

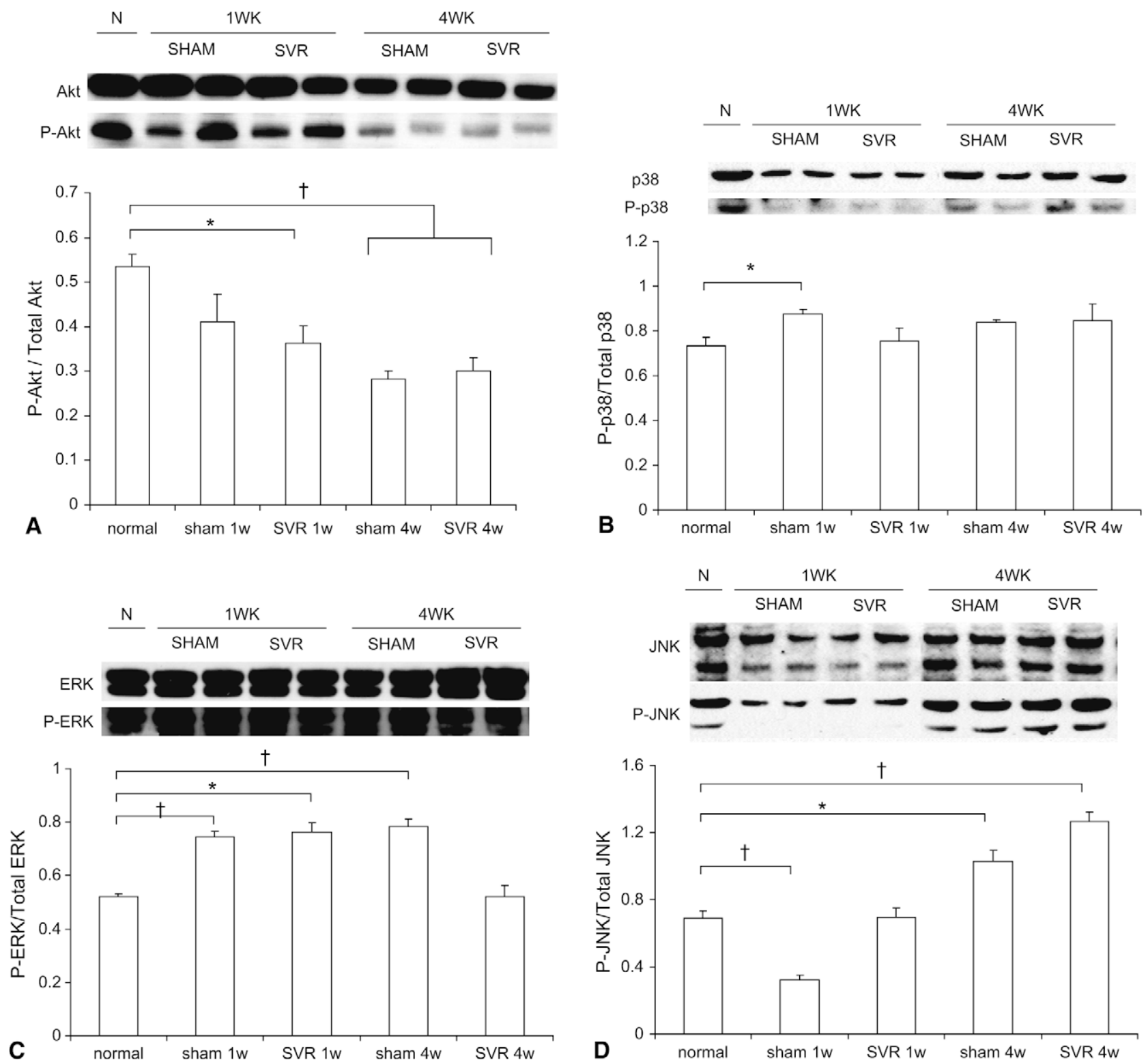

FIGURE 6. Myocardial signaling after SVR. Patterns of phosphorylation (activation) of Akt (A) and of MAP kinases p38 (B), ERK 1/2 (C), and JNK (D) at 1 and 4 weeks after reoperation in SVR and sham control hearts $(\dagger P<.01, * P<.05, \mathrm{n}=3-4)$. SVR, Surgical ventricular reconstruction. $M A P$, mitogen-activated protein.

through application of this model in genetic mouse models of diffuse coronary atherosclerosis. In addition, permanent coronary occlusion resulted in a large transmural infarction/aneurysm that exaggerates the typical infarctions of modern candidates for aneurysm resection. Future variations of this model, such as temporary ligation, may extend these observations toward a broader range of human clinical scenarios. The rapid progression of $\mathrm{LV}$ remodeling after $\mathrm{MI}$ in mice, and of recurrent dilatation after SVR, may require transition through larger animal models for accurate extrapolation to human translation.

If one key to recurrent $\mathrm{LV}$ dilatation, however, lies in the molecular biology of the cardiac myocyte, ${ }^{30}$ then the murine model described here may be well suited to identify critical molecular pathways in the response of the ventricle to SVR. The relative ease with which transgenic strains can be developed in mice has already yielded a wide array of available genetic models. Interestingly, the rapid progression of recurrent dilatation in mice may provide an advantage in the early discovery process. Ongoing studies have begun to examine SVR in the context of myocardial overexpression of activated Akt in transgenic mice. Data from these and other studies may, in turn, provide a foundation for the development of novel, hybrid surgical interventions for failing hearts, in which genetic, molecular, or cell-based therapies may be applied intraoperatively on the basis of a more 
detailed appreciation of the molecular and cellular parameters of ventricular recovery after reconstruction.

\section{References}

1. Redmann K, Lunkenheimer PP, Dietl KH, Cryer CW, Batista RJ, Anderson RH. Immediate effects of partial left ventriculectomy on left ventricular function. J Card Surg. 1998;13:453-62.

2. Mickleborough LL, Merchant N, Ivanov J, Rao V, Carson S. Left ventricular reconstruction: early and late results. J Thorac Cardiovasc Surg. 2004;128: 27-37.

3. Nishina T, Miwa S, Yuasa S, Nishimura K, Komeda M. A rat model of ischaemic or dilated cardiomyopathy for investigating left ventricular repair surgery. Clin Exp Pharmacol Physiol. 2002;29:728-30.

4. Athanasuleas CL, Buckberg GD, Stanley AW, Siler W, Dor V, Di Donato M, et al. Surgical ventricular restoration in the treatment of congestive heart failure due to post-infarction ventricular dilation. J Am Coll Cardiol. 2004;44: 1439-45.

5. Jackson BM, Gorman JH, Moainie SL, Guy TS, Narula N, Narula J, et al. Extension of borderzone myocardium in postinfarction dilated cardiomyopathy. $\mathrm{J} \mathrm{Am}$ Coll Cardiol. 2002;40:1160-7.

6. Battaloglu B, Erdil N, Nisanoglu V. Left ventricular aneurysmal repair within 30 days after acute myocardial infarction: early and mid-term outcomes. Tex Heart Inst J. 2007;34:154-9.

7. Chen FY, Cohn LH. The surgical treatment of heart failure. A new frontier: nontransplant surgical alternatives in heart failure. Cardiol Rev. 2002;10:326-33.

8. Tønnessen T, Knudsen CW. Surgical left ventricular remodeling in heart failure. Eur J Heart Fail. 2005; 7:704-9.

9. Sakaguchi G, Young RL, Komeda M, Yamanaka K, Buxton BF, Louis WJ. Left ventricular aneurysm repair in rats: structural, functional, and molecular consequences. J Thorac Cardiovasc Surg. 2001;121:750-61.

10. Selvetella G, Hirsch E, Notte A, Tarone G, Lembo G. Adaptive and maladaptive hypertrophic pathways: points of convergence and divergence. Cardiovasc Res. 2004;63:373-80.

11. McMullen JR, Amirahmadi F, Woodcock EA, Schinke-Braun M, Bouwman RD, Hewitt KA, et al. Protective effects of exercise and phosphoinositide 3-kinase (p110alpha) signaling in dilated and hypertrophic cardiomyopathy. Proc Natl Acad Sci U S A. 2007;104:612-7.

12. Oudit GY, Crackower MA, Eriksson U, Sarao R, Kozieradzki I, Sasaki T, et al. Phosphoinositide 3-kinase gamma-deficient mice are protected from isoproterenol-induced heart failure. Circulation. 2003;108:2147-52

13. Liao P, Georgakopoulos D, Kovacs A, Zheng M, Lerner D, Pu H, et al. The in vivo role of 338 MAP kinases in cardiac remodeling and restrictive cardiomyopathy. Proc Natl Acad Sci U S A. 2001;98:12283-8.

14. Petrich BG, Molkentin JD, Wang Y. Temporal activation of c-Jun $N$-terminal kinase in adult transgenic heart via cre-loxP-mediated DNA recombination. FASEB J. 2003;17:749-51.
15. Lips DJ, Bueno OF, Wilkins BJ, Purcell NH, Kaiser RA, Lorenz JN, et al. MEK1 ERK2 signaling pathway protects myocardium from ischemic injury in vivo. Circulation. 2004;109:1938-41.

16. Aikawa R, Nawano M, Gu Y, Katagiri H, Asano T, Zhu W, et al. Insulin prevents cardiomyocytes from oxidative stress-induced apoptosis through activation of PI3 kinase/Akt. Circulation. 2000;102:2873-9.

17. Sahn DJ, DeMaria A, Kisslo J, Weyman A. Recommendations regarding quantitation in M-mode echocardiography: results of a survey of echocardiographic measurements. Circulation. 1978;58:1072-83.

18. Kanno S, Lerner DL, Schuessler RB, Betsuyaku T, Yamada KA, Saffitz JE, et al. Echocardiographic evaluation of ventricular remodeling in a mouse model of myocardial infarction. J Am Soc Echocardiogr. 2002;15:601-9.

19. Nishina T, Nishimura K, Yuasa S, Miwa S, Nomoto T, Sakakibara Y, et al. Initia effects of the left ventricular repair by plication may not last long in a rat ischemic cardiomyopathy model. Circulation. 2001;104:I241-5.

20. Kramer CM, Magovern JA, Rogers WJ, Vido D, Savage EB. Reverse remodeling and improved regional function after repair of left ventricular aneurysm. J Thorac Cardiovasc Surg. 2002;123:700-6.

21. Dor V, Sabatier M, Di Donato M, Montiglio F, Toso A, Maioli M. Efficacy of endoventricular patch plasty in large postinfarction akinetic scar and severe left ventricular dysfunction: comparison with a series of large dyskinetic scars. J Thorac Cardiovasc Surg. 1998;116:50-9.

22. Suma H, Isomura T, Horii T, Sato T, Kikuchi N, Iwahashi K, et al. Nontransplant cardiac surgery for end-stage cardiomyopathy. J Thorac Cardiovasc Surg. 2000;119:1233-45.

23. Shiojima I, Walsh K. Regulation of cardiac growth and coronary angiogenesis by the Akt/PKB signaling pathway. Genes Dev. 2006;20:3347-65.

24. Clerk A, Cullingford TE, Fuller SJ, Giraldo A, Markou T, Pikkarainen S, et al. Signaling pathways mediating cardiac myocyte gene expression in physiological and stress responses. J Cell Physiol. 2007;212:311-22.

25. Kocher AA, Schuster MD, Szabolcs MJ, Takuma S, Burkhoff D, Wang J, et al Neovascularization of ischemic myocardium by human bone-marrow-derived an gioblasts prevents cardiomyocyte apoptosis, reduces remodeling and improves cardiac function. Nat Med. 2001;7:430-6.

26. Klevenberg S, Golub JS, Amit M, Itskovitz-Eldor J, Langer R. Endothelial cells derived from human embryonic stem cells. Proc Natl Acad Sci U S A. 2002;99:4391-6.

27. Miyahara Y, Nagaya N, Kataoka M, Yanagawa B, Tanaka K, Hao H, et al. Monolayered mesenchymal stem cells repair scarred myocardium after myocardial infarction. Nat Med. 2006;12:459-65.

28. Badylak SF, Kochupura PV, Cohen IS, Doronin SV, Saltman AE, Gilbert TW, et al. The use of extracellular matrix as an inductive scaffold for the partial replacement of functional myocardium. Cell Transplant. 2006;15:S29-40.

29. Callegari A, Bollini S, Iop L, Chiavegato A, Torregrossa G, Pozzobon M, et al. Neovascularization induced by porous collagen scaffold implanted on intact and cryoinjured rat hearts. Biomaterials. 2007;28:5449-61.

30. Nordlie MA, Wold LE, Simkhovich BZ, Sesti C, Kloner RA. Molecular aspects of ischemic heart disease: ischemia/reperfusion-induced genetic changes and potential applications of gene and RNA interference therapy. J Cardiovasc Pharmaco Ther. 2006;11:17-30. 